\title{
ON AN IDENTITY RELATED TO MULTIVALENT FUNCTIONS
}

\author{
A. Z. GRINSHPAN \\ (Communicated by Albert Baernstein II) \\ Dedicated to Professor A. W. Goodman on his 80th birthday
}

\begin{abstract}
We prove an algebraic identity by induction. This identity is very important in the coefficient problem for analytic functions that are $p$-valent in the unit disk.
\end{abstract}

\section{INTRODUCTION}

In 1948 Goodman [1] proposed the conjecture that if

$$
f(z)=\sum_{n=1}^{\infty} b_{n} z^{n}
$$

is regular and $p$-valent in $E:|z|<1$, then the coefficients satisfy the inequality

$$
\left|b_{n}\right| \leq \sum_{k=1}^{p} D(p, k, n)\left|b_{k}\right|, \quad 1 \leq k \leq p<n,
$$

where by definition

$$
D(p, k, n)=\frac{2 k(n+p) !}{(p+k) !(p-k) !(n-p-1) !\left(n^{2}-k^{2}\right)} .
$$

It was proved in [1] that if the conjecture were true it would be sharp for every selection of the $p$ variables $\left|b_{1}\right|,\left|b_{2}\right|, \ldots,\left|b_{p}\right|$. In a very nice paper Goodman and Robertson [2] proved that the conjecture (2) is true for the class $T(p)$, a very large class of multivalent functions. The class $T(p)$ is the natural extension of the Rogosinski class of typically-real functions to the class of typically-real functions of order $p$. Further, $T(p)$ includes the class of all $p$-valent starlike functions with real coefficients.

The proof in [2] depends on an algebraic identity symbolized by

$$
D^{*}(p, k, n)=D(p, k, n), \quad 1 \leq k \leq p<n,
$$

Received by the editors July 20, 1993.

1991 Mathematics Subject Classification. Primary 30C50, $30 \mathrm{C} 45$.

Key words and phrases. Multivalent functions, p-valent functions, Goodman's conjecture, typically-real functions of order $p$. 
where $D^{*}(p, k, n)$ will be defined later. The proof of (4) given in [2] is quite sophisticated and subtle, but can also be regarded as artificial and unsatisfactory because it is not direct. In this paper we give a direct proof of (4).

\section{THE ALGEBRAIC IDENTITY}

In [2], equation (3.17) in that paper, the quantities $D^{*}(p, k, n)$ are defined by equating the corresponding coefficients of $\left|c_{k}^{(p)}\right|$ on both sides of the equation

$$
\begin{aligned}
\sum_{k=1}^{p} D^{*}(p, k, n)\left|c_{k}^{(p)}\right|= & (n-p)\left|c_{p-1}^{(p)}\right|+(n-p+1)\left|c_{p}^{(p)}\right| \\
& +\sum_{j=p}^{n-1}(n-j) \sum_{s=1}^{p-1} D(p-1, s, j)\left[\left|c_{s+1}^{(p)}\right|+2\left|c_{s}^{(p)}\right|+\left|c_{s-1}^{(p)}\right|\right]
\end{aligned}
$$

where we set $c_{0}^{(p)}=0$.

In this equation the absolute value signs are not needed (for our purposes) and the superscripts in $c_{k}^{(p)}$ are unnecessary. Henceforth we drop both of these items.

Theorem. Equation (4) holds for every set of positive integers satisfying $1 \leq k \leq$ $p<n$.

\section{THE PROOF}

We first regroup the terms in (3.17) by changing the range of the indices. The standard technique will give

$$
\begin{aligned}
\sum_{k=1}^{p} D^{*}(p, k, n) c_{k}= & (n-p) c_{p-1}+(n-p+1) c_{p} \\
& +\sum_{j=p}^{n-1}(n-j) \sum_{s=2}^{p} D(p-1, s-1, j) c_{s} \\
& +\sum_{j=p}^{n-1}(n-j) \sum_{s=1}^{p-1} D(p-1, s, j) 2 c_{s} \\
& +\sum_{j=p}^{n-1}(n-j) \sum_{s=0}^{p-2} D(p-1, s+1, j) c_{s} .
\end{aligned}
$$

Equating coefficients in (5), will give $p$ equations but special attention must be given when $k=p$ or $k=p-1$. Further, when $p \geq 4$, one must consider three different cases. To avoid this difficulty we extend the definition of $D(p, k, n)$ in a natural way. Let

$$
\begin{gathered}
D(p, 0, j)=0 \quad \text { all } p, j, \\
D(p-1, p, j)= \begin{cases}1, & \text { if } j=p, \\
0, & \text { if } j>p,\end{cases}
\end{gathered}
$$




$$
D(p-1, p+1, j)= \begin{cases}0, & \text { if } j=p \\ -1, & \text { if } j=p+1 \\ 0, & \text { if } j>p+1\end{cases}
$$

Then (5), (6), (7) and (8) give

$$
\begin{aligned}
D^{*}(p, k, n)=\sum_{j=p}^{n-1}(n-j)[ & D(p-1, k-1, j) \\
& +2 D(p-1, k, j)+D(p-1, k+1, j)]
\end{aligned}
$$

for $1 \leq k \leq p<n$.

We use induction on $n$ to prove that (4) holds for all $n=p+1, p+2, \ldots$. For $n=p+1$, equation (9) gives

$$
\begin{aligned}
D^{*}(p, k, p+1) & =D(p-1, k-1, p)+2 D(p-1, k, p)+D(p-1, k+1, p) \\
& =\frac{2(2 p-1) !}{(p+k) !(p-k) !}\left[\frac{(k-1)(p+k)}{(p-k+1)}+2 k+\frac{(k+1)(p-k)}{(p+k+1)}\right] .
\end{aligned}
$$

The sum inside the brackets will give $\left(4 k p^{2}+2 k p\right) /\left[(p+1)^{2}-k^{2}\right]$, after a small computation. Hence $D^{*}(p, k, n)=D(p, k, n)$ when $n=p+1$.

Now assume that (4) is true for $n=p+1, p+2, \ldots, N$. Thus

$$
\begin{aligned}
D(p, k, n)=\sum_{j=p}^{n-1}(n-j)[ & D(p-1, k-1, j) \\
& +2 D(p-1, k, j)+D(p-1, k+1, j)]
\end{aligned}
$$

for $n=p+1, p+2, \ldots, N$. We must examine

$$
\begin{aligned}
D^{*}(p, k, N+1)=\sum_{j=p}^{N}(N+1-j)[ & D(p-1, k-1, j) \\
& +2 D(p-1, k, j)+D(p-1, k+1, j)] .
\end{aligned}
$$

We break the first factor into two parts, 1 and $N-j$, and observe that in the second sum we have 0 when $j=N$. Consequently

$$
\begin{aligned}
& D^{*}(p, k, N+1) \\
& =\sum_{j=p}^{N}[D(p-1, k-1, j)+2 D(p-1, k, j)+D(p-1, k+1, j)] \\
& \quad+\sum_{j=p}^{N-1}(N-j)[D(p-1, k-1, j) \\
& \quad+2 D(p-1, k, j)+D(p-1, k+1, j)] .
\end{aligned}
$$

By the induction hypothesis $(11)$, the second sum is $D(p, k, N)$, so

$$
\begin{aligned}
D^{*}(p, k, N+1) & \\
=D(p, k, N)+\sum_{j=p}^{N}[D(p-1, k-1, j) & +2 D(p-1, k, j) \\
& +D(p-1, k+1, j)] .
\end{aligned}
$$


To complete the proof, we do a second induction. In (12) we replace $N$ by $n-1$ and define $D^{* *}(p, k, n)$ by

$$
\begin{aligned}
D^{* *}(p, k, n) & \\
=D(p, k, n-1)+\sum_{j=p}^{n-1}[D(p-1, k-1, j) & +2 D(p-1, k, j) \\
& +D(p-1, k+1, j)] .
\end{aligned}
$$

We will use induction to prove that $D^{* *}(p, k, n)=D(p, k, n)$ for $n \geq p+2$ and hence conclude that $D^{*}(p, k, n)=D(p, k, n)$. We observe that with the natural extension $D(p, p, p)=1$. Further if $k=p$ and $n=p+1$ then (10) and (13) give $D^{* *}(p, p, p+1)=1+D(p, p, p+1) \neq D(p, p, p+1)$.

When $n=p+2$, equation (13) gives

$$
\begin{aligned}
D^{* *}(p, & k, p+2) \\
= & D(p, k, p+1) \\
& +D(p-1, k-1, p)+2 D(p-1, k, p)+D(p-1, k+1, p) \\
& +D(p-1, k-1, p+1)+2 D(p-1, k, p+1) \\
& +D(p-1, k+1, p+1) .
\end{aligned}
$$

From (10) the second line in (14) gives $D(p, k, p+1)$. Hence

$$
\begin{aligned}
D^{* *}(p, k, p+2)= & 2 D(p, k, p+1)+D(p-1, k-1, p+1) \\
& +2 D(p-1, k, p+1)+D(p-1, k+1, p+1) .
\end{aligned}
$$

Using the definition of $D(p, k, n)$ and dropping $0 !=1 !=1$, we have

$$
\begin{aligned}
D^{* *}(p, k, p+2)= & 2 \frac{2 k(2 p+1) !}{(p+k) !(p-k) !\left[(p+1)^{2}-k^{2}\right]} \\
& +\frac{2(k-1)(2 p) !}{(p+k-2) !(p-k) !\left[(p+1)^{2}-(k-1)^{2}\right]} \\
& +2 \frac{2 k(2 p) !(p-k)}{(p+k-1) !(p-k) !\left[(p+1)^{2}-k^{2}\right]} \\
& +\frac{2(k+1)(2 p) !(p-k)(p-k-1)}{(p+k) !(p-k) !\left[(p+1)^{2}-(k+1)^{2}\right]},
\end{aligned}
$$

or

$$
D^{* *}(p, k, p+2)=\frac{2(2 p) !}{(p+k) !(p-k) !}\left[s_{1}+s_{2}+s_{3}+s_{4}\right] .
$$

Here by definition,

$$
s_{1}+s_{3}=\frac{2 k(2 p+1)}{(p+1)^{2}-k^{2}}+\frac{2 k\left(p^{2}-k^{2}\right)}{(p+1)^{2}-k^{2}}=2 k,
$$

and

$$
\begin{aligned}
s_{2}+s_{4} & =\frac{(k-1)(p+k-1)}{p-k+2}+\frac{(k+1)(p-k-1)}{p+k+2} \\
& =\frac{2 k\left(p^{2}-p+k^{2}-3\right)}{(p+2)^{2}-k^{2}} .
\end{aligned}
$$


Thus $s_{1}+s_{2}+s_{3}+s_{4}=k(2 p+1)(2 p+2) /\left[(p+2)^{2}-k^{2}\right]$, and hence from (16)

$$
D^{* *}(p, k, p+2)=\frac{2 k(p+2) !}{(p+k) !(p-k) !\left[(p+2)^{2}-k^{2}\right]}=D(p, k, p+2) \text {. }
$$

We now apply mathematical induction to the statement that $D^{* *}(p, k, n)=$ $D(p, k, n)$ assuming that it is true for indices $n=p+2, p+3, \ldots, L$. From (13) we have

$$
\begin{aligned}
D^{* *}(p, k, L+1) & \\
=D(p, k, L)+\sum_{j=p}^{L} & {[D(p-1, k-1, j)} \\
& +2 D(p-1, k, j)+D(p-1, k+1, j)]
\end{aligned}
$$

or

$$
\begin{array}{r}
D^{* *}(p, k, L+1)=D(p, k, L)-D(p, k, L-1)+D(p, k, L-1) \\
+\sum_{j=p}^{L}[D(p-1, k-1, j)+2 D(p-1, k, j) \\
+D(p-1, k+1, j)] .
\end{array}
$$

But by the induction hypothesis

$$
\begin{aligned}
& D(p, k, L-1)+\sum_{j=p}^{L-1}[D(p-1, k-1, j)+2 D(p-1, k, j) \\
& +D(p-1, k+1, j)] \\
& =D(p, k, L) \text {. }
\end{aligned}
$$

So (21) becomes

$$
\begin{aligned}
D^{* *}(p, k, L+1)= & 2 D(p, k, L)-D(p, k, L-1)+D(p-1, k-1, L) \\
& +2 D(p-1, k, L)+D(p-1, k+1, L) \\
= & 2 \frac{2 k(L+p) !}{(p+k) !(p-k) !(L-p-1) !\left(L^{2}-k^{2}\right)} \\
& -\frac{2 k(L+p-1) !}{(p+k) !(p-k) !(L-p-2) !\left[(L-1)^{2}-k^{2}\right]} \\
& +\frac{2(k-1)(L+p-1) !}{(p+k-2) !(p-k) !(L-p) !\left[L^{2}-(k-1)^{2}\right]} \\
& +2 \frac{2 k(L+p-1) !(p-k)}{(p+k-1) !(p-k) !(L-p) !\left(L^{2}-k^{2}\right)} \\
& +\frac{2(k+1)(L+p-1) !(p-k)(p-k-1)}{(p+k) !(p-k) !(L-p) !\left[L^{2}-(k+1)^{2}\right]}
\end{aligned}
$$

The first and fourth terms in the above expression combine to give

$$
(2 k) \frac{2(L+p-1) !}{(p+k) !(p-k) !(L-p) !} \text {. }
$$

Then $D^{* *}(p, k, L+1)$ can be written as

$$
D^{* *}(p, k, L+1)=\frac{2(L+p-1) !}{(p+k) !(p-k) !(L-p) !} I(p, k, L),
$$


where

$$
\begin{aligned}
I(p, k, L)= & 2 k-\frac{k(L-p)(L-p-1)}{(L-1)^{2}-k^{2}} \\
& +\frac{(k-1)(p+k)(p+k-1)}{L^{2}-(k-1)^{2}} \\
& +\frac{(k+1)(p-k)(p-k-1)}{L^{2}-(k+1)^{2}} .
\end{aligned}
$$

To simplify $I(p, k, L)$ we use one $k$ and the next two terms in (24) and set

$$
\Phi(p, k, L)=k-\frac{k(L-p)(L-p-1)}{(L-1)^{2}-k^{2}}+\frac{(k-1)(p+k)(p+k-1)}{L^{2}-(k-1)^{2}} \text {. }
$$

Observing that the L.C.D. in $(25)$ is $\left[(L-k)^{2}-1\right](L+k-1)$, we have

$$
\begin{aligned}
\Phi(p, k, L) \\
=k+\frac{1}{L+k-1} \\
\quad \cdot\left(\frac{-k(L-p)(L-p-1)(L+1-k)+(k-1)(p+k)(p+k-1)(L-1-k)}{(L-k)^{2}-1}\right) .
\end{aligned}
$$

Let $\mathscr{N}$ be the numerator of the last term in $\Phi$. We write $\mathscr{N}$ as a polynomial in $p$ and after a moderate computation we find that

$$
\begin{aligned}
\mathscr{N}= & p^{2}(-L-k+1)+p(L+k-1)\left(2 L k-2 k^{2}+1\right) \\
& +k(L+k-1)\left(-L^{2}+2 k L-L+1-k^{2}\right) .
\end{aligned}
$$

After an obvious cancellation, this gives

$$
\begin{aligned}
\Phi(p, k, L) & =k+\frac{-p^{2}+\left(2 L k-2 k^{2}+1\right) p+k\left(-L^{2}+2 k L-L+1-k^{2}\right)}{(L-k)^{2}-1} \\
& =\frac{-p^{2}+\left(2 L k-2 k^{2}+1\right) p-k L}{(L-k)^{2}-1} .
\end{aligned}
$$

From this last expression it is clear that $\Phi(p, k, L)=\Phi(p,-k,-L)$ and using this in (25) we find that

(28) $\Phi(p,-k,-L)=-k+\frac{k(L+p)(L+p+1)}{(L+1)^{2}-k^{2}}-\frac{(k+1)(p-k)(p-k-1)}{L^{2}-(k+1)^{2}}$.

From (24) and (25) $I(p, k, L)$ can be put in the form

$$
I(p, k, L)=k+\Phi(p, k, L)+\frac{(k+1)(p-k)(p-k-1)}{L^{2}-(k+1)^{2}} .
$$

When $\Phi(p, k, L)$ is replaced by $\Phi(p,-k,-L)$ given by (28) we find that

$$
I(p, k, L)=\frac{k(L+p)(L+p+1)}{(L+1)^{2}-k^{2}} .
$$

Combining this with (23) we have

$$
\begin{aligned}
D^{* *}(p, k, L+1) & =\frac{2(L+p-1) !}{(p+k) !(p-k) !(L-p) !} \cdot \frac{k(L+p)(L+p+1)}{(L+1)^{2}-k^{2}} \\
& =D(p, k, L+1) .
\end{aligned}
$$




\section{REFERENCES}

1. A. W. Goodman, On some determinants related to p-valent functions, Trans. Amer. Math. Soc. 63 (1948), 175-192.

2. A. W. Goodman and M. S. Robertson, A class of multivalent functions, Trans. Amer. Math. Soc. 70 (1951), 127-136.

Department of Mathematics, University of South Florida, Tampa, Florida 33620

E-mail address: azg@math.usf.edu

Russian Academy of Sciences, INENCO, St. Petersburg, 190000, Russia 